\title{
An approach for classification of malignant and benign microcalcification clusters
}

\author{
BIRMOHAN SINGH ${ }^{1, *}$ and MANPREET KAUR ${ }^{2}$ \\ ${ }^{1}$ Department of Computer Science and Engineering, Sant Longowal Institute of Engineering and Technology, \\ Longowal 148106, India \\ ${ }^{2}$ Department of Electrical and Instrumentation Engineering, Sant Longowal Institute of Engineering and \\ Technology, Longowal 148106, India \\ e-mail: birmohans@gmail.com; aneja_mpk@yahoo.com
}

MS received 8 February 2016; revised 7 March 2017; accepted 21 August 2017; published online 16 March 2018

\begin{abstract}
The only reliable and successful treatment of breast cancer is its detection through mammography at initial stage. Clusters of microcalcifications are important signs of breast cancer. Manual interpretation of mammographic images, in which the suspicious regions are indicated as areas of varying intensities, is not error free due to a number of reasons. These errors can be reduced by using computer-aided diagnosis systems that result in reduction of either false positives or true negatives. The purpose of the study in this paper is to develop a methodology for distinguishing malignant microcalcification clusters from benign microcalcification clusters. The proposed approach first enhances the region of interest by using morphological operations. Then, two types of features, cluster shape features and cluster texture features, are extracted. A Support Vector Machine is used for classification. A new set of shape features based on the recursive subsampling method is added to the feature set, which improves the classification accuracy of the system. It has been found that these features are capable of differentiating malignant and benign tissue regions. To investigate the performance of the proposed approach, mammogram images are taken from Digital Database for Screening Mammography database and an accuracy of $94.25 \%$ has been achieved. The experiments have shown that the proposed classification system minimizes the classification errors and is more efficient in correct diagnosis.
\end{abstract}

Keywords. Microcalcifications; texture features; shape features; hierarchical centroid; Support Vector Machine.

\section{Introduction}

Breast cancer is the second leading cause of female cancer mortality; $16 \%$ of all the cancer cases in women are the breast cancer cases. The breast cancer cases in developed countries are very high and it is a serious cause of concern while in case of less developed countries the numbers of cases are continuously rising. The mortality rate is very high in developing countries as compared with developed countries. The low survival rates in developing countries may be due to lack of awareness and early detection [1]. The detection of breast abnormalities at the initial stage is important to increase the survival rate since it restricts a women from entering into further stages of breast cancer. Amongst all the imaging modalities available, mammography is the most reliable imaging modality to detect breast abnormalities at the early stage [2]. It uses low-dose amplitude $\mathrm{X}$-rays to examine the breast. Cancerous masses and calcium deposits appear brighter on the mammogram.

*For correspondence
Mammogram images give some indication to the radiologist in detecting the abnormal growth of cells. Mammography has helped decrease the mortality rate by $25-30 \%$ [3].

However, unfortunately, the success rate in the manual screening of the mammogram images is not very high. A number of factors, like human factors, poor quality of imaging, the number of benign cases in comparison to number of malignant cases, number of mammograms in comparison to number of radiologists, etc., are some of the causes for incorrect manual diagnosis [4]; $43 \%$ of errors are due to overlooking signs in abnormal scans and 52\% of the errors are due to misinterpretation of breast cancer signs [5].

Mass and microcalcifications are two main types of findings that indicate a possibility of cancer in the breast tissue at the early stage. Masses are lesions that may represent a localizing sign of breast cancer. Masses are dense regions and described by location, size, shape and margin characteristics. Microcalcifications are the accumulations of calcium and may show up in clusters or in patterns. They 
appear as bright spots on mammograms [6]. Detection of microcalcifications is difficult as they contain a variable number of glandular and fatty tissue that may decrease the visibility of subtle microcalcifications $[7,8]$.

The most important aspect to differentiate malignant microcalcifications from benign is the morphology, including size of calcification, number of calcifications in a cluster, etc. Large, coarse, round and smooth margin microcalcifications are benign. Microcalcifications that are fine, have linear boundaries and appear in clusters are malignant. Microcalcifications that are found in clusters have clinical significance [7]. Clustered microcalcifications are important signs of breast cancer at an earlier stage and appear in $30-50 \%$ of mammograms that are diagnosed. Only $15-34 \%$ of cases sent for biopsy are malignant and $10-30 \%$ of actual malignant cases are missed by radiologists [8]. Double reading by second radiologists can improve sensitivity of diagnosis, but the dearth of expert radiologists in comparison with the number of mammograms generated is the major problem.

Computer-aided diagnosis (CAD) systems are the feasible alternatives that can furnish a second opinion to radiologists for reducing interpretation errors, increasing the accuracy of diagnosis and improving the consistency of diagnosis. Two types of system exist: one deals with detecting and identifying the region of interest (ROI), while the other deals with classification of abnormality into benign or malignant $[4,9]$.

An efficient CAD system to classify microcalcifications clusters from the breast mammogram images is needed that can assist in correct identification of malignant clusters. In this study, the goal is to propose an approach to develop a system that identifies a ROI as malignant microcalcification or benign microcalcification cluster since the classification of microcalcification clusters is difficult due to their overlapping properties. The proposed methodology contributes in preprocessing and use for enhancement, providing a unique combination of features.

The rest of the paper is organized in sections. Section 2 presents literature review. Section 3 provides the details of database and datasets employed. Section 4 presents proposed methodology for classification of microcalcification clusters in detail. Section 5 demonstrates experimental results, related discussion and compares the classification results to the work of other researchers. Section 6 draws the conclusions of this work.

\section{Literature review}

The presence of microcalcification clusters in a mammogram indicates the primary sign of breast cancer. Manual screening by radiologists is difficult and time-consuming. Hence, many researchers have contributed in the development and the growth of CAD systems. The researchers who have explored the area to distinguish malignant and benign microcalcifications have either used only the texture features [10-17], or a combination of texture and shape features [4, 18-20]. For extracting the shape-based features, some researchers have worked to extract shape and topology of individual microcalcifications [21, 22]. These features become ineffective and do not provide much useful information when the microcalcifications are very small. Also, the microcalcifications may have low contrast due to their presence in the dense breast areas. This makes it difficult to analyse the shape of individual microcalcifications. Moreover, it may not provide any distinct texture features for such areas. Hence, some other researchers have extracted the shape features for a cluster of microcalcifications [4, 19, 23].

Microcalcifications are characterized based on their appearance, characteristics and distribution. Malignant microcalcifications are generally smaller, denser and more irregular than benign microcalcifications. A microcalcification cluster is an area of $1 \mathrm{~cm}^{2}$ with no fewer than 5 microcalcifications [17]. The distribution of microcalcifications associated with a malignant structure is different from that of a benign structure. Malignant microcalcifications are densely distributed than benign because they lie within the milk ducts and associated structures. Hence, these variations in the distribution and closeness of microcalcifications within a cluster provide useful information. Moreover, the tissue texture of microcalcification cluster associated with a malignant region may be different from that associated with a benign region. Considering this as motivation, many texture models in combination with shape-based feature models have been studied and analysed so that a better combination of these features may improve the classification rate to discriminate malignant and benign microcalcification clusters.

\section{Database}

To evaluate and test the proposed system the images collected from Image Retrieval in Medical Applications (IRMA) version of Digital Database for Screening Mammography (DDSM) dataset have been used. One of the four scanners, DBA M2100 ImageClear $(42 \mu \mathrm{m}$ per pixel, 16 bits), Howtek 960 (43.5 $\mu \mathrm{m}$ per pixel, 12 bits), Lumisys 200 Laser $(50 \mu \mathrm{m}$ per pixel, 12 bits) and Howtek MultiRad850 (43.5 $\mu \mathrm{m}$ per pixel, 12 bits), was used to digitize a mammograms image from DDSM database [24].

For classification of microcalcifications, 273 malignant and 155 benign image patches are taken. These patches have been manually identified and are annotated by expert radiologists. Three set of images are constituted. Set1 consists of 80 mammogram regions of interest of size $256 \times 256$ pixels with 40 malignant and 40 benign microcalcifications. Set 2 contains 130 (79 malignant and 51 
Table 1. Set of images for classification of microcalcification clusters.

\begin{tabular}{lccc}
\hline Image set & Total & Malignant & Benign \\
\hline Set1 & 80 & 40 & 40 \\
Set2 & 130 & 79 & 51 \\
Set3 & 218 & 154 & 64 \\
\hline
\end{tabular}

benign) square regions of $256 \times 256$ pixels and Set 3 contains 218 (154 malignant and 64 benign) rectangular regions of different sizes. Set1 is used for training purpose and other sets are used for testing the proposed methodology. The details of these sets are given in table 1 .

\section{Proposed methodology}

A system has been proposed to classify microcalcification clusters into malignant or benign. The proposed system is based on two types of features: cluster shape features and cluster texture features. The block diagram of the proposed system is shown in figure 1. A region of suspicion for microcalcifications is extracted manually from a mammogram image. This region is enhanced by using morphological operations. Individual microcalcifications are identified and cluster is marked. For this cluster region, the shape of the cluster is identified and based on this shape, the cluster features are extracted. In order to extract texture features, a minimum region corresponding to cluster shape is used. A feature matrix is formed for these features that are reduced and input to a classifier to distinguish benign and malignant microcalcification clusters.

One of the suspicious regions with microcalcification cluster is extracted from Mammogram image D_4118_1.RIGHT_MLO (figure 2a). This region is further enhanced by the enhancement procedure discussed in the next section. Some of the regions enhanced with the proposed methodology are shown in figure 3. To identify a cluster, the region is converted into a binary image (figure $2 \mathrm{~b}$ ) and the boundary of the cluster is marked as shown in figure 2c. The corresponding shape of the cluster is shown in figure $2 \mathrm{~d}$, which is used for extracting the shapebased features. For extracting the texture features, a minimum cluster area is marked (figure $2 \mathrm{e}$ ) and this region is selected for texture features.

\subsection{Enhancement of microcalcifications}

Microcalcifications are difficult to detect if they are present in the dense breast area. A proper enhancement method can remove noise and improve contrast of an image. Various techniques have been proposed and adopted to enhance mammogram images by researchers. Most commonly used

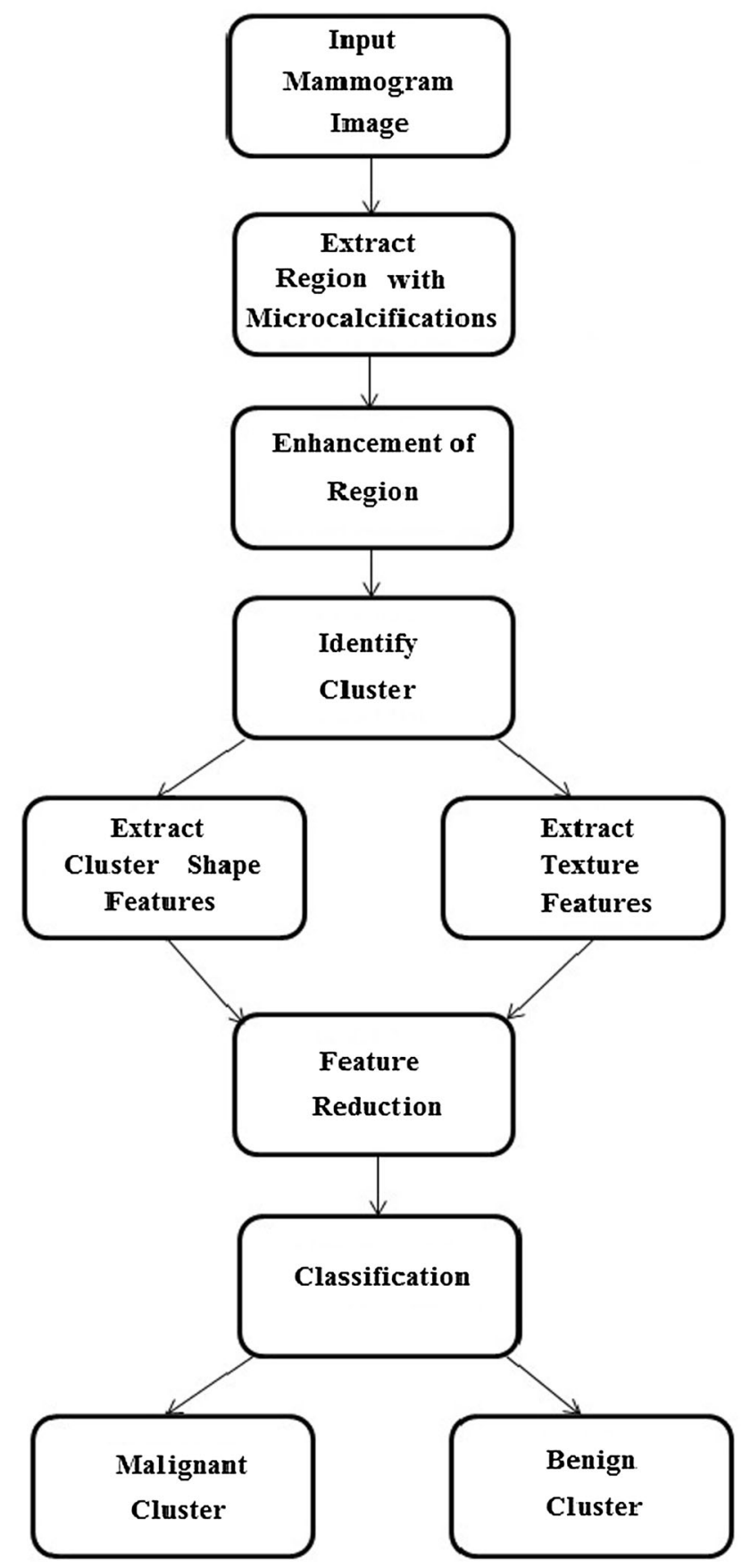

Figure 1. Block diagram of proposed computer aided classification system for microcalcifications.

techniques are histogram equalization, unsharp masking, wavelet decomposition, region-based methods, fuzzy set methods and fractal modelling [4].

The purpose of enhancement is to improve contrast of microcalcifications clusters with respect to the background. Histogram modification methods, spatial domain and frequency domain filtering, and mathematical morphologybased methods are the most common enhancement techniques for mammogram images. However, these methods 
enhance the entire image without discrimination, whereas for effective detection of region, it is necessary to enhance only specific target regions and not the surrounding tissues [25]. Researchers have used several morphological contrast enhancement methods for enhancement of microcalcifications in mammograms [26-29]. The proposed method improves the contrast of microcalcification regions solely, while the background region is suppressed.

In this paper, ROI is enhanced with morphological operations. Let $\mathrm{F}$ be an input mammogram, $\mathrm{S}$ be the structuring element and $\mathrm{C}$ be the complement of the structuring element.

$$
\begin{gathered}
F 1=F-(F o S) \\
F 2=(F \bullet S)-F \\
F 3=F-(F o C) \\
F 4=(F \bullet C)-F \\
F 5=F 1+F 3 \\
F 6=F 2+F 4 \\
E I=(F+F 5)-F 6
\end{gathered}
$$

where $\mathrm{o}$ and $\bullet$ represent morphological opening and closing of an image, respectively. EI is the enhanced image. The structuring element used is

\begin{tabular}{|l|l|l|l|l|l|l|}
\hline 0 & 0 & 0 & 1 & 0 & 0 & 0 \\
\hline 0 & 1 & 1 & 1 & 1 & 1 & 0 \\
\hline 0 & 1 & 1 & 1 & 1 & 1 & 1 \\
\hline 1 & 1 & 1 & 1 & 1 & 1 & 1 \\
\hline 1 & 1 & 1 & 1 & 1 & 1 & 0 \\
\hline 0 & 1 & 1 & 1 & 1 & 1 & 0 \\
\hline 0 & 0 & 0 & 1 & 0 & 0 & 0 \\
\hline
\end{tabular}

Structuring element of $7 \times 7$ yielded the best results as compared with $3 \times 3$ and $5 \times 5$, and similar results as compared to $9 \times 9$, when enhancement results were inspected visually. The performance of images enhanced with structuring elements of $3 \times 3,5 \times 5,7 \times 7$ and $9 \times 9$ is shown in figure 3 .

\subsection{Segmentation of cluster}

The purpose of image segmentation is to divide an image into non-overlapping regions formed on the basis of image intensity or image texture. After image enhancement, there is a need to identify the cluster of microcalcifications, for each of the enhanced regions. The aim is to compute several discriminating features for classifying malignant regions from benign regions. Different techniques have been adopted by researchers for image segmentation like edge detection, region growing, histogram thresholding, stochastic models, clustering models, etc. [30]. It has been observed that mathematical morphological operations like erosion, top hat transformations and filters have been widely used for segmentation [4, 19, 31, 32].

In this work, a method has been proposed for marking the clusters. It has been assumed that there exists only one cluster in a ROI. Sine transformation is applied to the enhanced image for separating the microcalcifications from the other breast regions. The sine transformation increases the high-intensity pixels further while retaining low-intensity pixels. The resultant image (RES) is formed by sine transformation from the enhanced image with the following equation:

$$
R E S=E I+\sin (E I)
$$

where sin returns the circular sine of the elements of EI. The sine function operates element-wise on EI. The resultant image is converted into a binary image. A cluster from binary image is formed by including all the individual microcalcifications. The line segments joining all the centroids of individual microcalcifications give the shape of cluster and this cluster region has been separated out to find the cluster shape properties. In order to extract texture region a minimum rectangular region of ROI has been considered in place of the whole ROI (figure 2f). The proposed feature extraction procedure is a hybrid procedure that combines, cluster-based shape features and clusterbased texture features.

\subsection{Feature extraction}

Different types of feature models have been proposed by researchers to classify microcalcification clusters into benign and malignant. Shape-based features are not significant in describing the exact shape of the individual microcalcifications when they are small in size. On the other hand texture features do not give useful information when microcalcifications are present in dense tissue with homogenous intensity [56]. Hence, the features that have been considered in this paper are shape features and texture features for cluster regions since the texture information as well as morphological information is considered important for classification of microcalcification clusters. The features extracted have a correlation with the factors describing a distinction between malignant and benign abnormalities.

4.3a Shape features: Texture features can be used to distinguish the normal and abnormal tissues, but these features are not very effective in classifying malignant lesions [33]. Hence, a combination of shape- and texture-based features has been used in this work. Morphology is one of the important factors for a diagnosis of microcalcification cluster. Considering cluster as one object, various shapebased features have been extracted, which describe cluster 

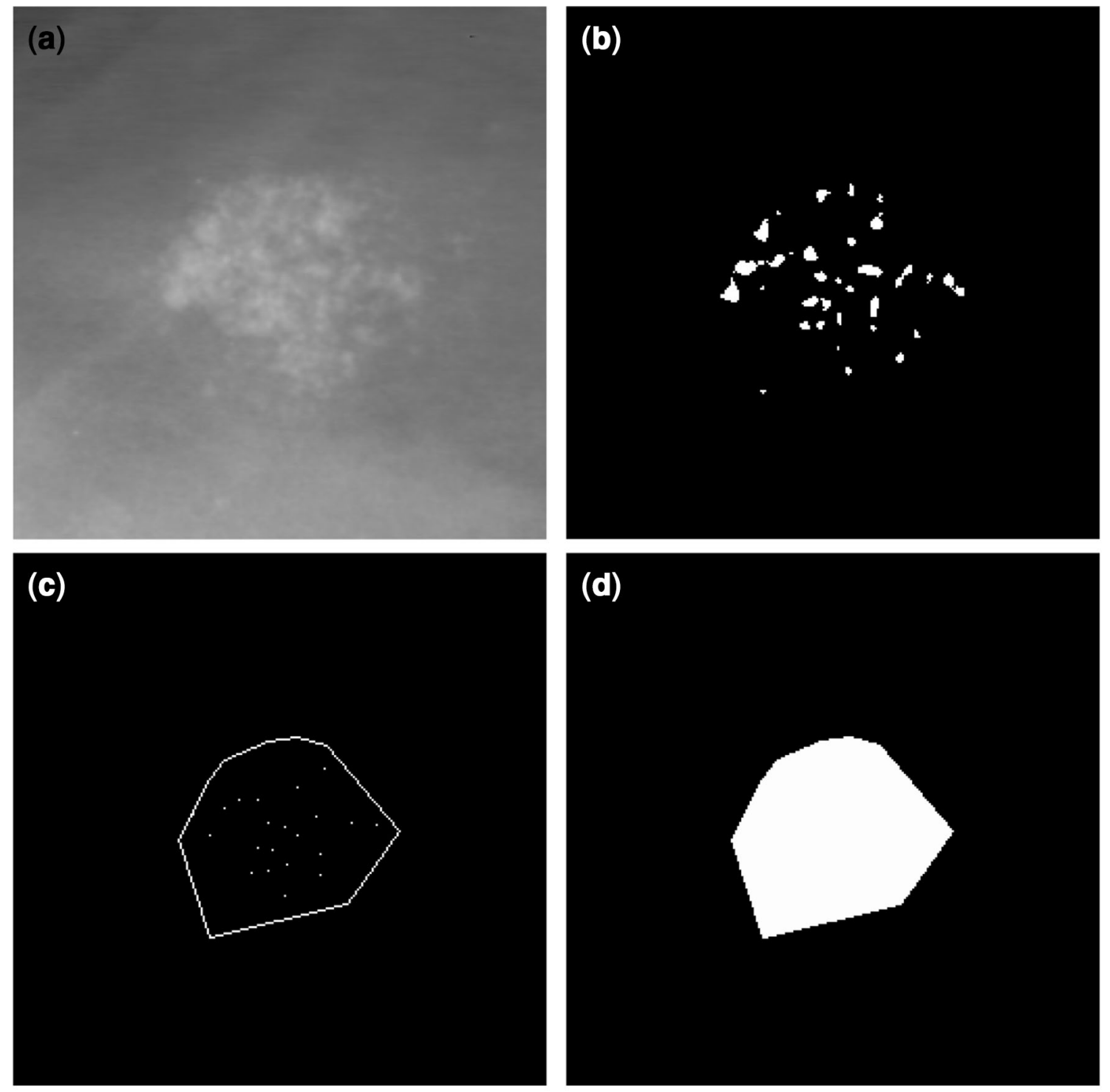

(e)

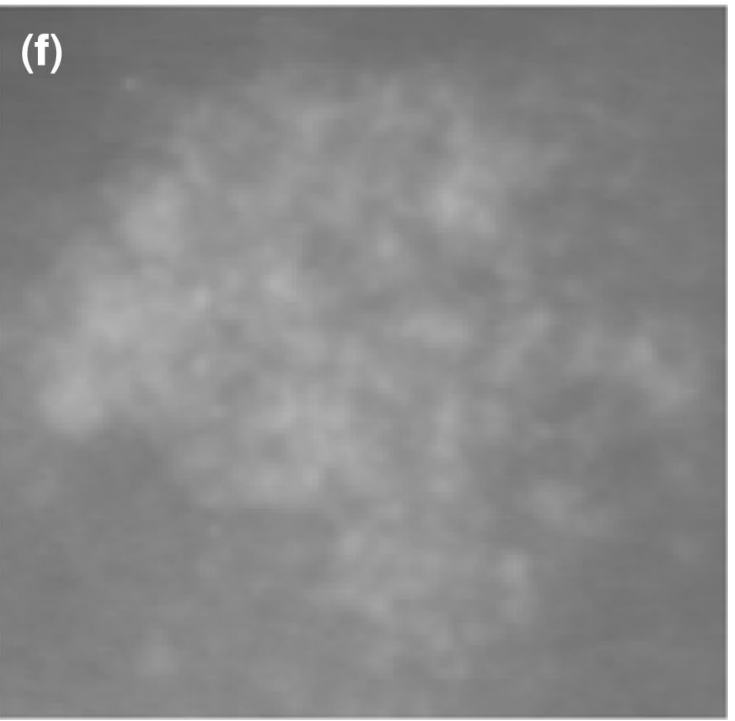

Figure 2. For ROI from D_4118_1.RIGHT_MLO (a) Original region. (b) Binary region. (c) Boundary of microcalcification cluster. (d) Shape of cluster for shape features. (e) Region corresponding to cluster. (f) Extracted corresponding region for texture features. 


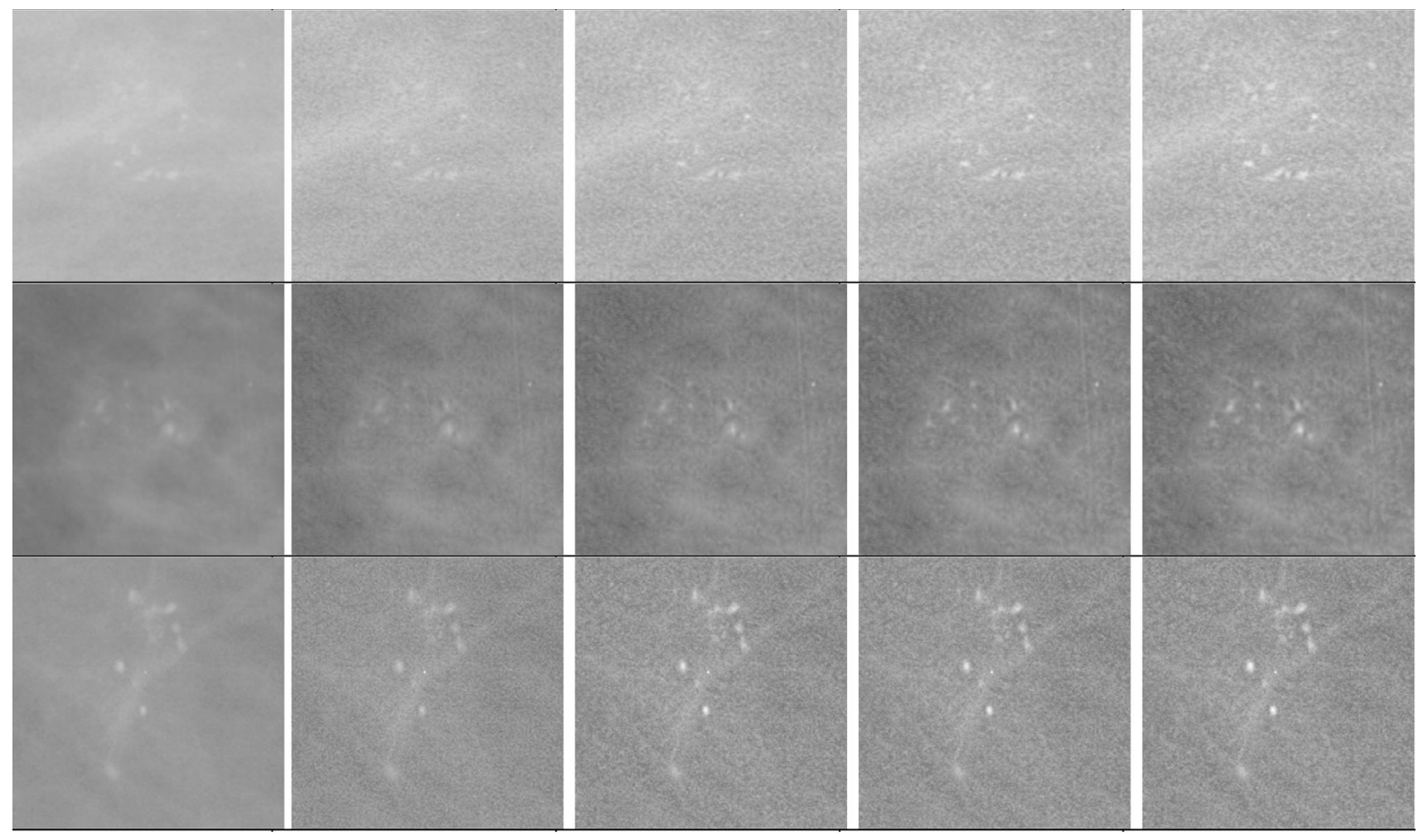

(a)

(b)

(c)

(d)

(e)

Figure 3. (a) Original image and enhanced images with structuring element of (b) $3 \times 3$, (c) $5 \times 5$, (d) $7 \times 7$, and (e) $9 \times 9$.

morphology. Shape features provide additional information about the object; hence, some geometrical features have been considered [4].

Malignant or benign microcalcification clusters are not described by some predefined shape patterns. Shape, curvature and size are part of the basic characteristics that can help in classifying clusters. Malignant clusters are more likely to be irregular, granular, fine linear and are of small size. Benign clusters have smooth boundary popcorn-like, large rod-like, round, lucent-centred and eggshell, and are of large size [18].

In all, 14 shape-based features have been extracted for each cluster as listed in table 2 (which shows features extracted and features selected). The feature reduction using Differential-Evolution-based Feature Selection (DEFS) method has been discussed in section 4.4. These features include number of microcalcifications in a cluster, area of cluster measured as number of pixels in that region, maximum diameter measured as length of the major axis of the ellipse that has the same normalized second central moments as those of the region, minimum diameter of cluster measured as length of the minor axis of the ellipse that has the same normalized second central moments as those of the region, perimeter measured as distance between each adjoining pair of pixels around the border of the region, extent computed as ratio of pixels in the region to pixels in the total bounding box, eccentricity calculated as ratio of the distance between the foci of the ellipse (that has the same second moments as those of the region) to its major axis length, orientation measured as angle (in degrees ranging from $-90^{\circ}$ to $90^{\circ}$ ) between the $x$-axis and the major axis of the ellipse that has the same second moments as those of the region, solidity, which specifies the proportion of the pixels in the convex hull that are also in the region, equivalent diameter, which is specified as the diameter of a circle with the same area as that of the region, density computed as ratio of area of microcalcifications to total area of the cluster, compactness computed as square of perimeter to area of cluster, mean and standard deviation of distances of pixels in the cluster from the centre.

Apart from these features, 28 new shape features extracted by the hierarchical centroid method [34], which is based on the recursive subsampling method [35] of image, have been proposed to be added to other shape-based features.

For a binary image of size $m \times n$, subsampling of the image is performed by iterative subdivision of the image in such a way that in each subdivision the numbers of foreground and background pixels are approximately balanced. The original image is first subdivided into sub-images by a vertical line that further results into four image segments by a line drawn horizontally in such a way that the absolute 
difference in the foreground and background pixels is minimum in each of the subdivisions either horizontally or vertically. The pixel at the intersection of the two lines is referred to as the division point (DP). At each iteration step, sub-image obtained in the previous step is further divided into four sub-images using the same procedure. Iterations are levels of granularity $(L)$, and at level $L$, the co-ordinates of all DPs are stored as features.

Hence, for every $L$, the dimensional feature vector is extracted. The larger the $L$, the better the representation of the region obtained, but this increases computation overheads [35]. Thus, 28 features based on the hierarchical centroid method are extracted. Best results for the hierarchical centroid method are achieved with values of granularity $(L)$ considered equal to 4 .

4.3b Texture features: Texture is a major component to represent the properties to distinguish the texture and it exists for a region rather than a pixel or a point. Analysis of texture proves to be important in many applications, especially medical imaging, and medical image processing [36]. To distinguish benign and malignant microcalcifications, various researchers have proposed different approaches to compute texture features extracted from a region that contains microcalcifications. The most commonly used texture features are GLCM-based texture features [10, 13, 19], SGLD-based texture features [11], GLRLMbased texture features [10], LBP-based texture features [15], Gabor texture features [9, 14] and wavelet-based texture features $[19,20]$.

In this paper different texture features are extracted and evaluated for each cluster region. The texture features that have been extracted in this work are Haralick texture, Laws texture, fractal texture, Adaptive Local Binary Patterns (ALBP) features and Statistical Feature Matrix (SFM) texture.

Haralick texture [37] measures are based on secondorder statistics of grey level histograms, which is accomplished with the help of GLCM. The features are calculated for distance $(d) 1-4$ and orientation $0^{\circ}, 45^{\circ}, 90^{\circ}$ and $135^{\circ}$. Thus, for each suspected region, 208 texture features have been extracted. Haralick features $1-52$ correspond to the features for $d=1$ and orientation $0^{\circ}, 45^{\circ}, 90^{\circ}$ and $135^{\circ}$. Haralick features 53-104 correspond to features for $d=2$ and orientation $0^{\circ}, 45^{\circ}, 90^{\circ}$ and $135^{\circ}$, and similarly the features $105-156$ for $d=3$ and features $157-208$ for $d=4$. To decrease the computational complexity, the performance of these features has been checked by considering distances separately; it has been observed experimentally that $d=1$ gives better performance than $d=2, d=3$ and $d=4$. Thus, 52 features have been extracted for $d=1$ and orientations $0^{\circ}$, $45^{\circ}, 90^{\circ}$ and $135^{\circ}$. The details of Haralick features extracted and the corresponding features selected for the angles mentioned for $d=1$ have been shown in table 3 .

Laws texture features [38] are used in many applications and are computed by convolving the image with a number of small masks. To detail the microstructures of textures, Laws texture features are extracted with convolution masks of $5 \times 5$, where masks are a combination of basic mnemonics corresponding to Level (L5), Edge (E5), Spot (S5), Wave (W5) and Ripple (R5). Texture features have been extracted by filtering the images with each one of 25 Laws masks or kernels (table 4). Averaging the filtered images, 14 texture energy (TE) images are formed. For these $14 \mathrm{TE}$ images, one first-order statistic that is the average intensity of image has been considered, thereby resulting in 14 Laws texture features. Laws texture array contains 14 output values in the sequence EE, SS, WW, RR, EL, SL, WL, RL, SE, WE, RE, WS, RS and RW.

Fractals measure geometric complexity to describe spatial patterns of texture. Fractals refer to complex patterns that occur at various scales. Fractal dimensions and fractal signatures are significant in texture analysis [39]. Based on the multi-resolution fractal dimensions, a new set of features has been proposed by authors of [40], which is also been called a set of fractal features (FF). Using this, two Hurst coefficients have been extracted for each cluster region.

Local Binary Patterns (LBP) are greyscale-invariant texture measures derived from a general definition of texture in a local neighbourhood. LBP combines characteristics of statistical and structural texture analysis. Estimation errors of local differences have been reduced in ALBP and are relatively insensitive to variations in illuminations [41]. ALBP texture features have been extracted for each cluster region.

Apart from this, features based on SFM [36] have been extracted. Four visual-perceptual features, coarseness, contrast, periodicity and roughness have been extracted for each cluster region.

Table 2. Details of shape features.

\begin{tabular}{lcc}
\hline $\begin{array}{l}\text { Sl. } \\
\text { no. }\end{array}$ & Shape features extracted & Selected \\
\hline 1 & Number of calcifications & Yes \\
2 & Area of cluster & Yes \\
3 & Compactness & Yes \\
4 & Density & No \\
5 & Maximum diameter of cluster & Yes \\
6 & Minimum diameter of cluster & No \\
7 & Perimeter & Yes \\
8 & Mean of all calcification distances from center & No \\
9 & Std. dev. of all calcification distances from & Yes \\
& center & \\
10 & Extent & Yes \\
11 & Eccentricity & No \\
12 & Orientation & Yes \\
13 & Solidity & Yes \\
14 & Equivalent diameter & No \\
\hline
\end{tabular}


Table 3. Details of Haralick features.

\begin{tabular}{lcc}
\hline S1. no. & Extracted Haralick features & Features selected \\
\hline 1. & Energy & $0^{\circ}, 45^{\circ}, 135^{\circ}$ \\
2. & Contrast & $45^{\circ}, 135^{\circ}$ \\
3. & Correlation & $90^{\circ}, 135^{\circ}$ \\
4. & Sum of variance & $0^{\circ}, 45^{\circ}$ \\
5. & Inverse difference moment & $0^{\circ}, 45^{\circ}, 90^{\circ}$ \\
6. & Sum average & $0^{\circ}, 135^{\circ}$ \\
7. & Sum variance & $45^{\circ}, 90^{\circ}$ \\
8. & Sum entropy & $0^{\circ}, 45^{\circ}$ \\
9. & Entropy & $0^{\circ}, 45^{\circ}, 90^{\circ}, 135^{\circ}$ \\
10. & Difference variance & $0^{\circ}, 90^{\circ}$ \\
11. & Entropy difference & $0^{\circ}, 45^{\circ}, 90^{\circ}, 135^{\circ}$ \\
12. & Info measure of correlation I & $0^{\circ}, 90^{\circ}$ \\
13. & Info measure of correlation II & $90^{\circ}, 135^{\circ}$ \\
\hline
\end{tabular}

Table 4. Details of Law's texture features.

\begin{tabular}{lcc}
\hline $\begin{array}{l}\text { S1. } \\
\text { no. }\end{array}$ & Law's texture features extracted & Features selected \\
\hline 1. & Average intensity of the image with & Average intensity \\
& EE, SS, WW, RR, EL, SL, WL, RL, & of the image \\
& SE, WE, RE, WS, RS and RW & with \\
& EE, WW, EL, SL, \\
& WL, RL, RS and \\
& RW \\
\hline
\end{tabular}

\subsection{Feature reduction}

Feature reduction is to remove redundant features from high-dimensional feature sets. A good feature selection method reduces computational cost, increases its classification accuracy and improves result comprehensibility [42]. Generally, Genetic Algorithms (GA), Sequential Forward Selection (SFS) Method, Sequential Backward Selection (SBS) Method, Principal Component Analysis (PCA), Independent Component Analysis (ICA) and Fisher Discriminant Analysis (FDA) are some of the techniques used by researchers.

A feature set is prepared by collecting all cluster shape and cluster texture features. Some of these features may be redundant, which may affect the performance of classifier as well as increase the complexity. In this paper, the DEFS method has been used for dimensionality reduction and 145 features have been selected, which yield the best output. Differential-evolution-based algorithms do global optimized searching and do lead to nesting effects. These algorithms do better optimal optimization than the other methods [43]. The numbers of control parameters, in differential-evolution-based methods, are very few and space complexity is also less [44]. DEFS is a relatively novel optimization technique to solve numerical optimization problems and has been used by various researchers in different types of problems [45-47].

In the DEFS method, the researchers presented a procedure to overcome the problem of dimensionality. They presented a selection method that utilized a combination of the differential evolution optimization and proposed a repair mechanism based on feature distribution measures. The probabilities of a combination of features were checked to give an optimum set. This was used to help the selection of the wavelet packet transform that was best suited for classification [48].

The DEFS method does not select the appropriate number of features automatically, so the number of suitable features has to be selected. The approach that has been adopted is based on selection of minimum and maximum number of features. Features are extracted from minimum to maximum range with a fixed step. The features that yield better accuracy are selected. Again, accuracy is checked for these features to finalize those features that result in the best accuracy. A pseudo-code for procedure to select features with DEFS method is shown here:

Input: Training and test data, No. of features, Desired no. of features, Population Size

Output: Selected No. of features

a) Set MIN, for minimum number of features

b) Set MAX, for maximum number of features

c) Repeat for set $=$ MIN to MAX step 10

i. Select feature[set] with DEFS method

ii. Compute accuracy [set] with SVM for feature[set]

d) Select highest value of accuracy[k] and corresponding feature[k] such that $\mathrm{k} \in$ set and MIN $\leq \mathrm{k} \leq \mathrm{MAX}$

e) Repeat for subset $=k-10$ to $k+10$ step 1

i. Select feature[subset] with DEFS method

ii. Compute accuracy [subset] with SVM for feature[subset]

f) Select feature $[\mathrm{m}]$ with the highest value of accuracy[m] such that $\mathrm{k}-10 \leq \mathrm{m} \leq \mathrm{k}+10$

In this work, 115 features have been extracted for each suspicious region. The desired numbers of features are selected for this feature set in a two-phase process using the DEFS method. In the first phase, features are computed from 25 to 95 with a gap of 10 with the Naïve Bayes method and for 100 iterations. Classification accuracy has been computed for all these 8 sets of features. A set with 70 features gave the best performance out of these 8 sets. Hence, in the next phase, all the features are computed from 66 to 74 . From this new subset, a feature set with 71 features yielded the best accuracy. The summary of features extracted and features selected are given in table 5.

From a set of 14 shape-based features, 9 features have been selected. Apart from these, 15 features have been selected from hierarchical centroid features. These features 
Table 5. Set of features used in classification.

\begin{tabular}{cccc}
\hline S1. no. & Feature & Total features & Features selected \\
\hline 1 & Shape based & 14 & 9 \\
2 & Hierarchical centroid & 28 & 15 \\
3 & Haralick texture & 52 & 32 \\
4 & Laws texture & 14 & 08 \\
5 & Fractal texture & 02 & 02 \\
6 & ALBP texture & 01 & 01 \\
7 & SFM texture & 04 & 04 \\
\hline
\end{tabular}

are in correlation with the shape and morphology of clusters. Out of the 52 Haralick-based texture features, 32 features have been selected. These features have been used by most of the researchers in detection as well as classification of microcalcifications [13, 19]. In all, 8 Laws texture features have been selected. These features measure intensity of TE images formed, which is different for malignant and benign clusters. Hurst coefficients give approximation of fractal dimension for mammogram images. Hurst coefficients in fractal features discriminate texture surface on the basis of roughness. Roughness is different for malignant and benign microcalcification regions. Hurst's coefficients are more useful in fatty regions. LBP performs better in dense breasts. There are variations in continuity of benign and malignant microcalcifications. Benign regions represent uniform LBP patterns, which have limited discontinuity as compared with malignant regions. SFM texture model is useful to describe surface textures. These features are very sensitive to the grey level variations in the image. Small variations in the texture of benign and malignant regions have reflections on these features.

\subsection{Classification}

Various classifiers have been proposed for classification of microcalcification clusters into malignant and benign. Most of these classifiers are of supervised type. Support Vector Machines (SVMs) $[4,5,8]$, Artificial Neural Networks [14], K-Nearest Neighbours [11], Bayesian classifiers [49], decision tree [5], Self-Adaptive Resource Allocation Network (SRAN) [9], etc. are some of the classifiers used in detection and diagnosis. The classification problem considered in this research is a two-class pattern classification that the output is either malignant or benign. Performance of SVM for unknown data is satisfactory as shown by many researchers and hence selected. SVM is a procedure based on structural learning theory that minimizes generalization error. The performance of SVM was evaluated for linear, Gaussian and polynomial kernels with testing variations of cost parameter $C$ and kernel parameters. The best output is yielded by SVM with linear kernel function and Sequential Minimal Optimization (SMO) method.

\section{Results and discussion}

For training and testing, the proposed diagnosis system mammogram images are taken from DDSM database. The region of suspicion is manually extracted from a mammogram image. The region extracted from the mammogram is enhanced with morphological operations.

It has been observed that microcalcifications are difficult to detect due to complex background. Enhancement algorithms may enhance undesired objects in the background like vessels or glands. The procedure proposed improves the contrast of microcalcifications but removes undesired objects as well. The image profile method [29] has been used to check the improvement of foreground objects. For a region, image profile along a straight line has been taken from two places: one where there exists more number of microcalcifications, and the other where there is less probability of microcalcifications. Figure 4 shows two regions for which profiles are taken along the line indicated and are plotted. The plot of image profile shows original intensity values as well as intensity values after enhancement by the proposed method. It is shown that enhancement highlights probable microcalcification locations and increases the contrast of these locations while there is no enhancement of the background region and the contrast of such areas remains approximately the same after enhancement.

Similarly, another profile along the region of normal area is taken for these two images. Plots of image profile showing original intensity values and intensity values after enhancement are shown in figure 5. It is shown that there is no enhancement of the background region and the contrast of such areas remains more or less the same after enhancement.

Further, the quantitative performance analysis was performed based on metrics of contrast evaluation, Peak Signal to Noise Ratio (PSNR), Absolute Mean Brightness Error (AMBE), Edge-Content-based contrast metric (EC) and Michelson law measure of enhancement by entropy (AMEE).

PSNR indicates a smaller difference between the original and enhanced image. This metric is the most widely used to measure quality of resultant image [50, 51]. AMBE is a measure of change in the global appearance of the image by computing the deviation of the mean intensity of the enhanced image from the mean intensity of the original image. For better results, lower values of AMBE are desired [52]. EC accumulates the contrast changes, giving a quantitative measure for enhancement of the contrast achieved. This gives an objective measure of detail enhancements and their higher value corresponds to the 

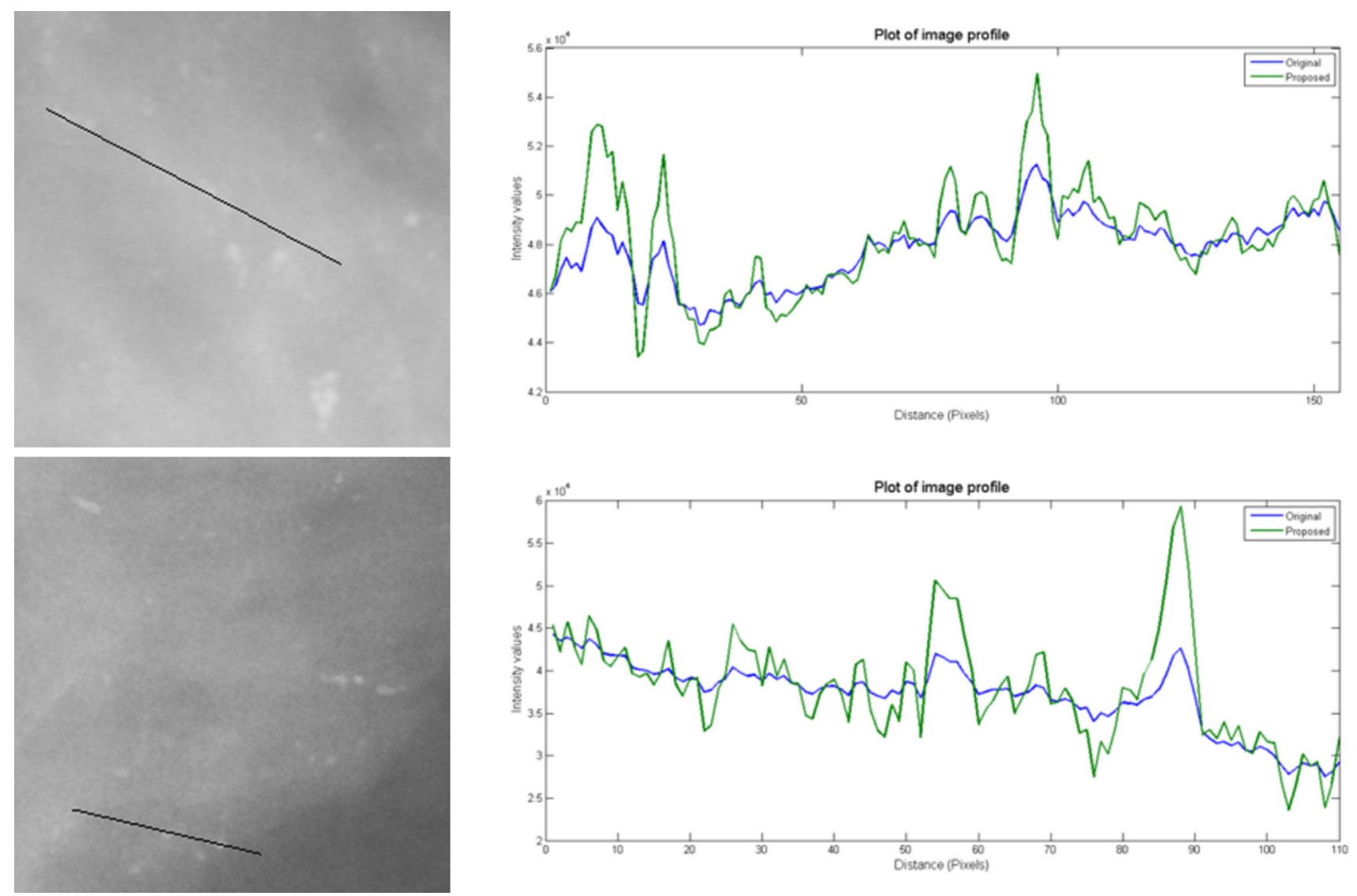

Figure 4. Image profile in the region with microcalcifications and the corresponding plot.

histogram equalized image [52]. The mammogram images tend to be textured and have a fair amount of randomness in the target area of the image. This attribute aligns with the properties of entropy-base measures, which can handle images with randomness and texture better. Hence, use of AMEE measure of image quality for mammograms has been recommended [53].

A comparison of PSNR for Histogram Equalization (HE) method, Contrast-Limited Adaptive Histogram Equalization (CLAHE) method and proposed method based on morphological operations has been shown in table 6 for some ROI extracted from DDSM database. A comparison of AMBE for these ROIs is shown in table 7 and EC is presented in table 8 . Table 9 shows a comparison of AMEE values for HE, CLAHE and the proposed method, for block size of $20 \times 20$ pixels and value of alpha as 0.1 . It has been observed that the proposed method for enhancement has performed better.

After enhancement of the regions with microcalcification clusters, they are converted into binary images (figure 6). From these images, the shape of the cluster region is marked by connecting the edge pixels in outer microcalcifications. Based on the shape, shape-based features are computed. The bounding box to this shape gives the minimum texture area and texture features are computed for this area. Figure 7 a shows binary images; the corresponding cluster shape is marked in figure $7 \mathrm{~b}$ and figure $7 \mathrm{c}$ shows image subdivision by the hierarchical centroid method.

A reduced set of optimal features, extracted from cluster shape and cluster texture, is input to SVM for classifying cluster regions into malignant or benign. The SVM classifier model built with training set is used to predict the output of testing sets. The performance of the proposed diagnosis system is evaluated with performance evaluation parameters that are commonly used in medical applications like sensitivity, specificity, accuracy and area under ROC curve (Az or AUC).

A classification system results a false positive (FP) if the system labels a negative case to a positive case, false negative (FN) if the system labels a positive case to a negative case $(\mathrm{FN})$, true positive (TP) and true negative (TN) if the system predicts the label correctly [53]. 

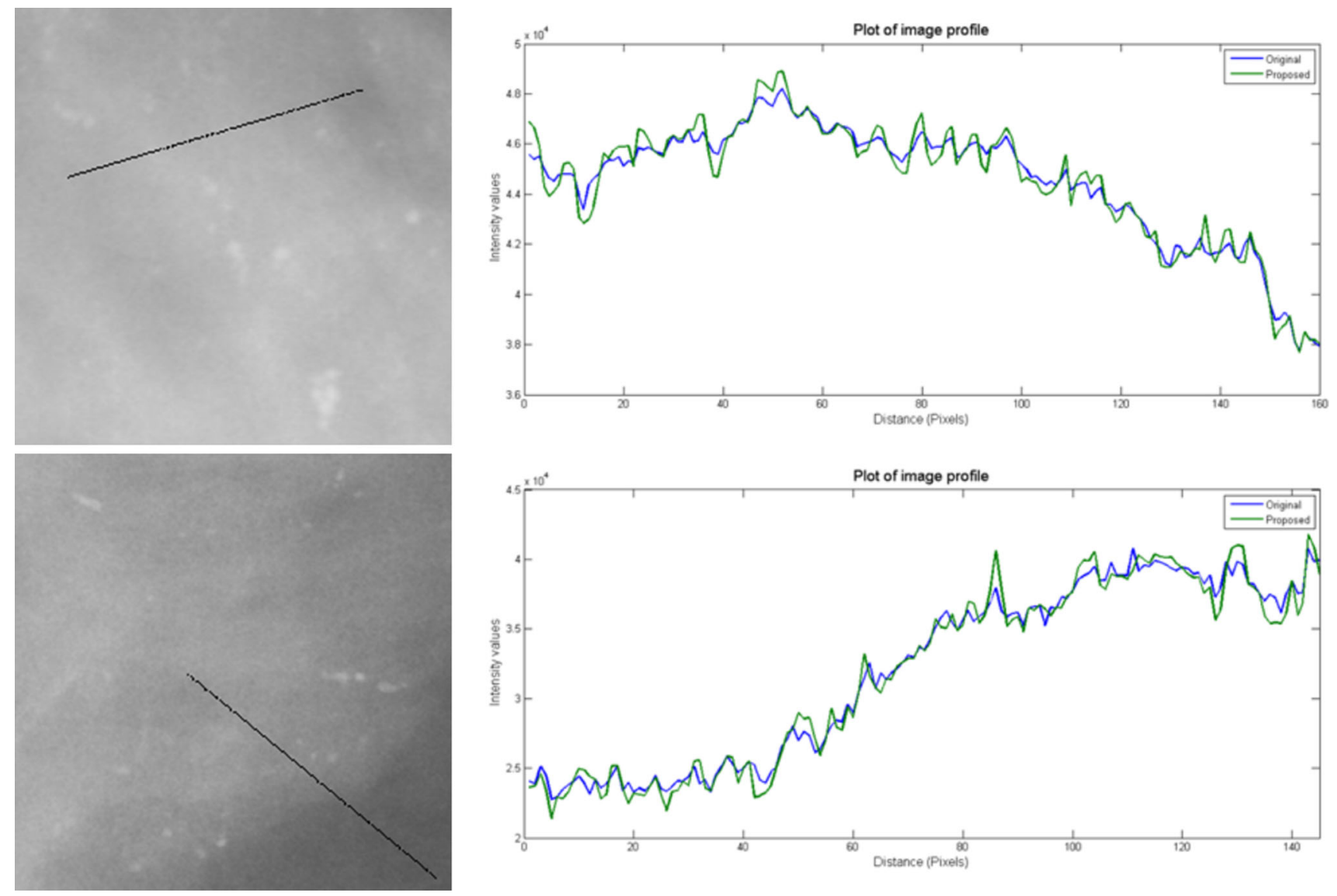

Figure 5. Image profile in the background region and the corresponding plot.

In this proposed diagnosis system, TP is taken as number of malignant images classified as malignant, TN value is number of benign images correctly identified, FP is number of benign images classified as malignant and FN is malignant images classified as benign. The output of SVM is compared to the original class attribute to get the values of TP, TN, FP and FN.

Sensitivity (Se) indicates the positive labels that are being truly classified and specificity (Sp) indicates the negative labels that are being truly classified by the classifier. Accuracy (Acc) is a measure to show how effectively positive and negative labels are correct in classification. Higher values of sensitivity and specificity are expected from a good classifier [54]. The other parameters that have been considered are positive predictive value (PPV), negative predictive value (NPV) and Youden's index. PPV indicates that abnormality is present when the result is positive. NPV indicates that the disease is not present when the test is negative. Youden's index indicates the ability to avoid failure. Higher values of these indices are preferred [55]. Receiver Operating Characteristic (ROC) curve analysis provides tools to select possibly optimal models and to discard suboptimal ones. It plots classification results from the most positive classification to the most negative classification [54].

The performance of the classification system for Set2 and Set 3 has been shown in table 10. It has been observed that Set2, which consists of square ROI, performs slightly better than Set3, which consists of rectangular regions of varying sizes. It can be concluded that the proposed system performs well both for suspicious regions of square size $256 \times 256$ pixels as well as for rectangular regions of any size. To check the response of the system, all the images from Set 2 and Set 3 have been combined to form Set 4 . All malignant regions except 8 are correctly classified, sensitivity is $96.57 \%$ and accuracy is $94.25 \%$. The average values of PPV, NPV and Youden's index for testing sets are $0.9494,0.9279$ and 0.8613 , respectively. Area under a receiver operating characteristic curve (AUC) for Set2, Set 3 and Set 4 are $0.9383,0.9258$ and 0.9307 , respectively.

The proposed methodology is validated and checked using $K$-fold cross validation procedure. $K$-fold cross-validation has been applied to the dataset to check parameter values of sensitivity, specificity, accuracy, PPV and NPV. The value of $K$ is varied from 5 to 12 and the results shown 


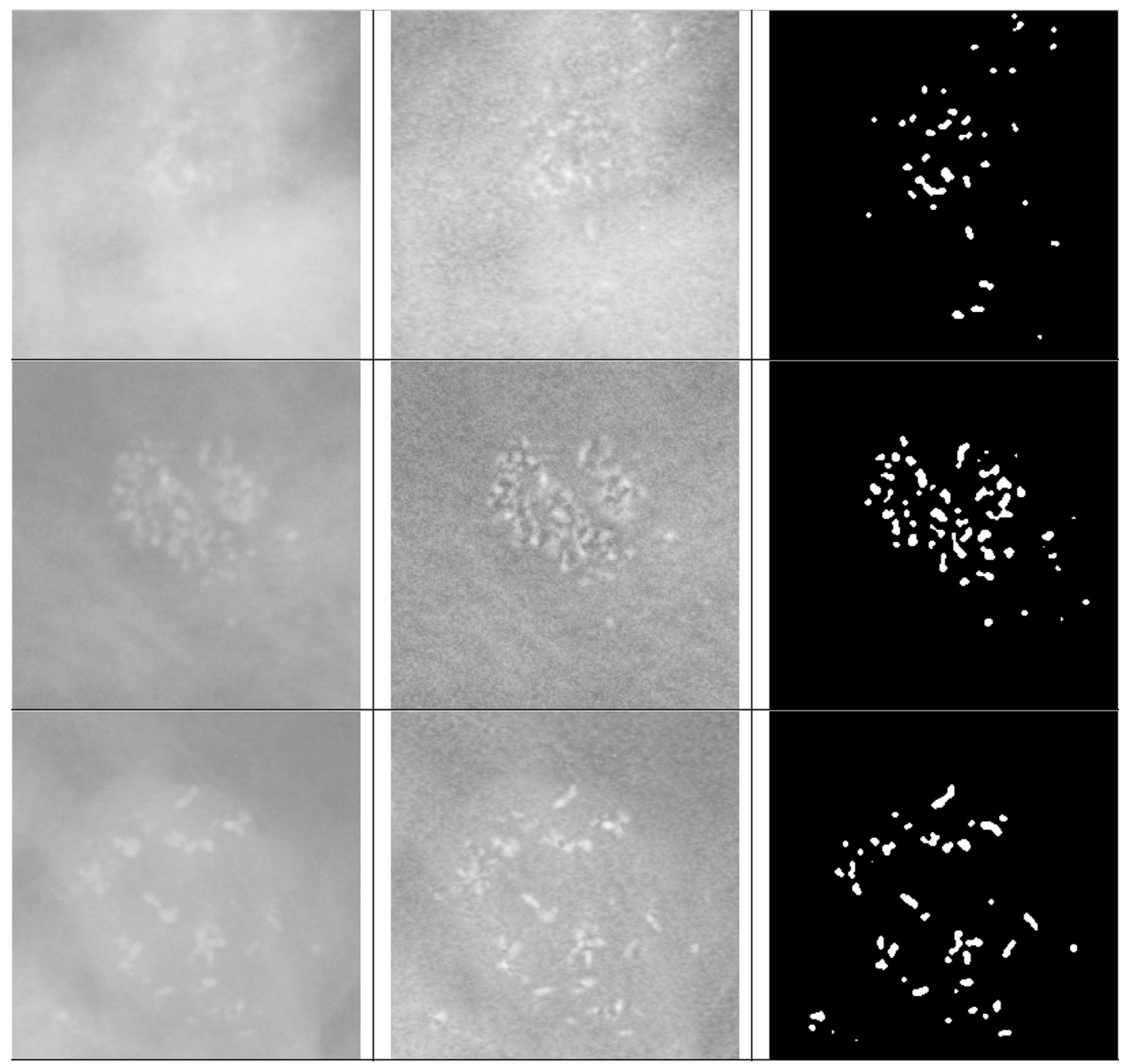

(a)

(b)

(c)

Figure 6. (a) Original image. (b) Enhanced image. (c) Binary image.

Table 6. PSNR for some regions extracted from DDSM.

\begin{tabular}{lccc}
\hline Image & HE & CLAHE & Proposed \\
\hline C_0106_1.LEFT_MLO & 58.4803 & 75.6056 & 79.5648 \\
A_1850_1.RIGHT_CC & 60.0497 & 74.7241 & 80.9637 \\
A_1894_1.RIGHT_MLO & 58.9177 & 77.7478 & 83.6822 \\
A_1906_1.LEFT_CC & 57.1266 & 77.4348 & 84.3075 \\
A_2000_1.RIGHT_MLO & 59.7120 & 74.6883 & 82.0559 \\
B_3060_1.RIGHT_CC & 59.1082 & 73.3462 & 79.7949 \\
D_4117_1.LEFT_CC & 58.6364 & 76.4780 & 81.9159 \\
D_4147_1.LEFT_CC & 59.5113 & 77.4472 & 85.1725 \\
D_4158_1.LEFT_MLO & 58.4538 & 76.5753 & 82.9423 \\
D_4190_1.RIGHT_CC & 59.1168 & 76.9143 & 81.6329 \\
D_4193_1.LEFT_MLO & 62.9104 & 73.3570 & 82.2182 \\
D_4200_1.RIGHT_CC & 58.9035 & 77.0496 & 83.7920 \\
\hline
\end{tabular}

in table 11 are averages of 10 iterations. Best results are achieved at $K=10$, having an accuracy of $94.48 \%$ with sensitivity $95.76 \%$ and specificity $93.75 \%$ for Set 4 .

The analysis of features proposed is also presented. The hierarchical centroid-based features have been proposed to be added in the cluster shape features set. ALBP features are included in the texture feature set. The performance of features is evaluated separately for all the ROIs and is summarized in table 12 .

From the experimental results, it has been observed that only cluster shape or only Haralick texture features or their combination are not sufficient for effective classification results. The accuracy of the system has increased from $72.70 \%$ to $87.36 \%$ when Laws, fractal and other texture features (coarseness, contrast, periodicity and roughness) 
Table 7. AMBE for some regions extracted from DDSM.

\begin{tabular}{lccc}
\hline Image & HE & CLAHE & Proposed \\
\hline C_0106_1.LEFT_MLO & 0.1530 & 0.0155 & 0.0005 \\
A_1850_1.RIGHT_CC & 0.0040 & 0.0102 & 0.0002 \\
A_1894_1.RIGHT_MLO & 0.1515 & 0.0093 & 0.0001 \\
A_1906_1.LEFT_CC & 0.2325 & 0.0151 & 0.0001 \\
A_2000_1.RIGHT_MLO & 0.1399 & 0.0213 & 0.0004 \\
B_3060_1.RIGHT_CC & 0.1523 & 0.0266 & 0.0001 \\
D_4117_1.LEFT_CC & 0.1377 & 0.0134 & 0.0002 \\
D_4147_1.LEFT_CC & 0.0970 & 0.0060 & 0.0002 \\
D_4158_1.LEFT_MLO & 0.1625 & 0.0179 & 0.0003 \\
D_4190_1.RIGHT_CC & 0.0633 & 0.0077 & 0.0005 \\
D_4193_1.LEFT_MLO & 0.0314 & 0.0239 & 0.0003 \\
D_4200_1.RIGHT_CC & 0.1036 & 0.0200 & 0.0001 \\
\hline
\end{tabular}

Table 8. EC for some regions extracted from DDSM.

\begin{tabular}{lrrr}
\hline Image & \multicolumn{1}{c}{$\mathrm{HE}$} & CLAHE & Proposed \\
\hline C_0106_1.LEFT_MLO & 58.8118 & 96.3811 & 120.2692 \\
A_1850_1.RIGHT_CC & 57.5173 & 86.4948 & 108.9398 \\
A_1894_1.RIGHT_MLO & 75.4928 & 106.8939 & 139.2245 \\
A_1906_1.LEFT_CC & 55.1244 & 111.0340 & 135.1656 \\
A_2000_1.RIGHT_MLO & 84.1668 & 96.4676 & 125.4928 \\
B_3060_1.RIGHT_CC & 75.8328 & 92.6799 & 119.2417 \\
D_4117_1.LEFT_CC & 55.0442 & 94.1932 & 119.5227 \\
D_4147_1.LEFT_CC & 70.0381 & 110.5149 & 139.0193 \\
D_4158_1.LEFT_MLO & 59.4270 & 92.8542 & 117.0755 \\
D_4190_1.RIGHT_CC & 54.2252 & 98.6233 & 116.6115 \\
D_4193_1.LEFT_MLO & 113.6033 & 98.2050 & 136.6367 \\
D_4200_1.RIGHT_CC & 55.2444 & 116.5465 & 139.4533 \\
\hline
\end{tabular}

Table 9. AMEE for some regions extracted from DDSM.

\begin{tabular}{lcccc}
\hline Image & Original & HE & CLAHE & Proposed \\
\hline C_0106_1.LEFT_MLO & 0.0851 & 0.0199 & 0.0590 & 0.0610 \\
A_1850_1.RIGHT_CC & 0.0770 & 0.0224 & 0.0502 & 0.0550 \\
A_1894_1.RIGHT_MLO & 0.0884 & 0.0333 & 0.0645 & 0.0696 \\
A_1906_1.LEFT_CC & 0.0945 & 0.0260 & 0.0704 & 0.0754 \\
A_2000_1.RIGHT_MLO & 0.0829 & 0.0343 & 0.0591 & 0.0648 \\
B_3060_1.RIGHT_CC & 0.0824 & 0.0291 & 0.0555 & 0.0595 \\
D_4117_1.LEFT_CC & 0.0864 & 0.0173 & 0.0602 & 0.0657 \\
D_4147_1.LEFT_CC & 0.0883 & 0.0345 & 0.0652 & 0.0712 \\
D_4158_1.LEFT_MLO & 0.0885 & 0.0224 & 0.0635 & 0.0688 \\
D_4190_1.RIGHT_CC & 0.0864 & 0.0109 & 0.0606 & 0.0627 \\
D_4193_1.LEFT_MLO & 0.0733 & 0.0479 & 0.0515 & 0.0557 \\
D_4200_1.RIGHT_CC & 0.0924 & 0.0230 & 0.0695 & 0.0711 \\
\hline
\end{tabular}

are included in texture features. The ALBP feature, which has been added to texture features, improves the accuracy to $1.3 \%$. There is an improvement of $6.1 \%$ when the proposed hierarchical centroid features are added to cluster shape features. The overall sensitivity and specificity of the system are, respectively, $96.57 \%$ and $89.57 \%$. The performance of ALBP and hierarchical centroid features is shown in figure 8.

A comparison of the proposed work is done with earlier work carried out by other researchers. A direct comparison however is not a simple task since different researchers have used different mammography databases, datasets, features and classifiers. Hence, a comparison on the basis of sensitivity, specificity, accuracy and AUC is prepared for the authors who have worked on the DDSM dataset, in table 13.

In 2007, authors of [10] suggested that texture analysis of tissues surrounding microcalcifications contributes in CAD of breast cancer. They extracted textural features (firstorder statistics, co-occurrence matrices features, run length matrices features and Laws' TE measures) and they achieved sensitivity $94.4 \%$ and specificity $80.0 \%$ for the mammograms collected from DDSM database. In 2013, authors of paper [20] used mammograms from MIAS as well as from DDSM database. They extracted seven features based on cluster shape (cluster maximum diameter, the cluster minimum diameter, the number of microcalcifications, the cluster area, the cluster perimeter, the cluster compactness and eccentricity). The sensitivity achieved was $93.5 \%$ and accuracy was $94.2 \%$. In 2014, authors of work [12] proposed a method based on mereotopological barcode analysis and achieved an accuracy of $80 \%$. In 2015, authors of [56] proposed a method based on topological structures and achieved an accuracy of $86 \%$ and area under ROC curve of 0.91 .

Shape-based features do not successfully describe the morphological properties when the size of individual microcalcifications is only a few pixels. When microcalcifications are present in dense breast tissue areas, where intensity is homogenous and contrast is low, texture features do not provide much useful information. Hence, a combination of shape and texture features has been proposed and it has improved the classification performance of the system. The overall accuracy achieved is $94.25 \%$ and AUC is 0.9307 . The results achieved with the proposed system show an improvement in classification performance over that of the other researchers.

\section{Conclusions}

In this paper, the objective is to propose an approach for classification of ROI that contains microcalcification cluster as malignant or benign and then to compare the performance with that of the work of other researchers, since the microcalcifications are difficult to detect, from the complex background. In the first step, ROI is enhanced 


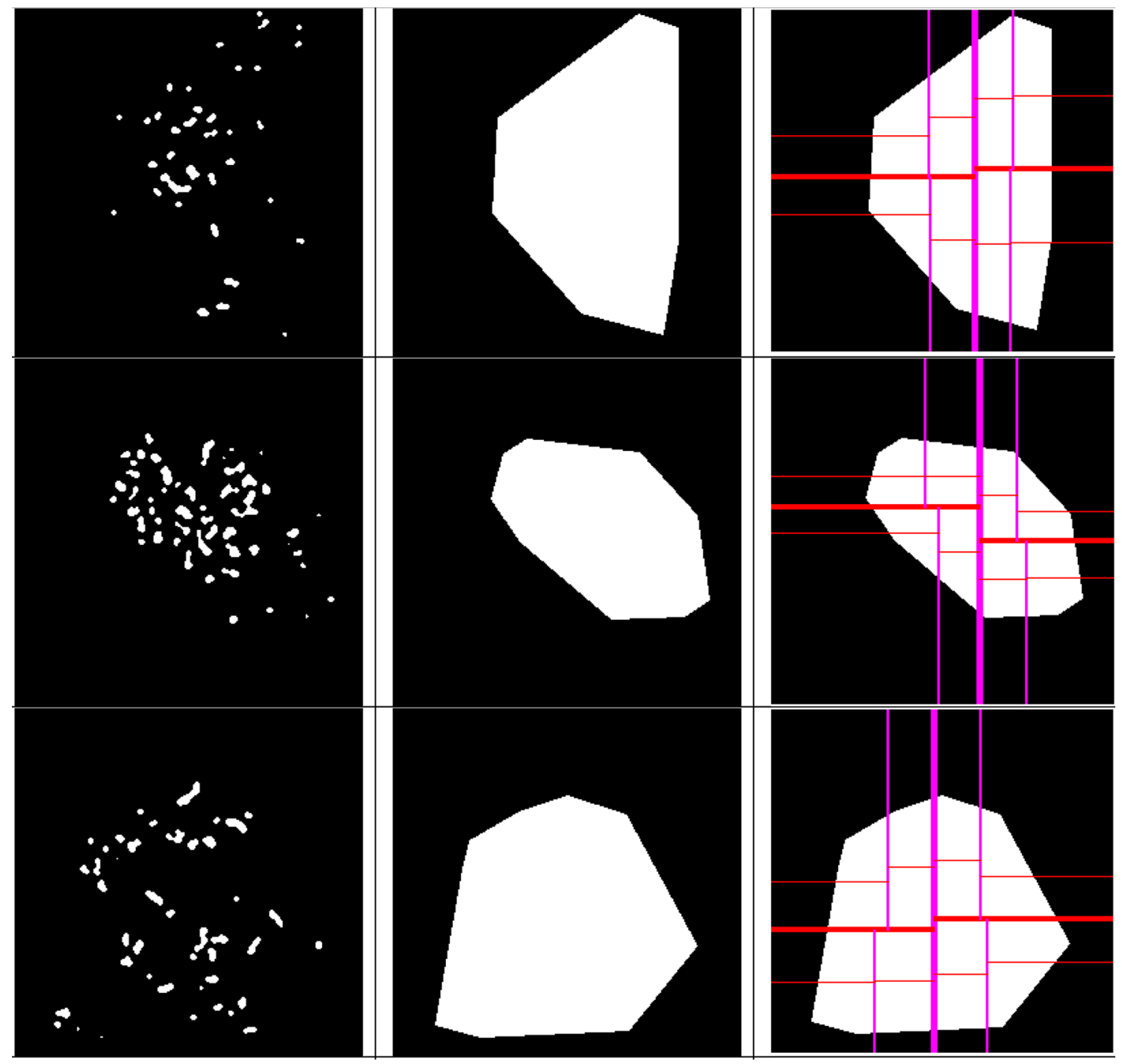

(a)

(b)

(c)

Figure 7. (a) Binary image. (b) Cluster shape. (c) Image subdivision with hierarchical centroid.

Table 10. Performance evaluation parameters of image sets.

\begin{tabular}{lrrrrcccccc}
\hline Image sets & TP & TN & FP & FN & Sensitivity & Specificity & Accuracy & PPV & NPV & Youden's index \\
\hline Set 2 & 77 & 46 & 5 & 2 & 0.9747 & 0.9020 & 0.9462 & 0.9390 & 0.9583 & 0.8766 \\
Set 3 & 148 & 57 & 7 & 6 & 0.9610 & 0.8906 & 0.9404 & 0.9548 & 0.9048 & 0.8517 \\
Set 4 & 225 & 103 & 12 & 8 & 0.9657 & 0.8957 & 0.9425 & 0.9494 & 0.9279 & 0.8613 \\
\hline
\end{tabular}


Table 11. Performance evaluation parameters using $K$-fold cross-validation.

\begin{tabular}{cccccc}
\hline $\begin{array}{l}K \text { value } \\
\text { of cross- } \\
\text { validation }\end{array}$ & Sensitivity & Specificity & Accuracy & PPV & NPV \\
\hline 5 & 0.9190 & 0.9286 & 0.9251 & 0.8808 & 0.9534 \\
6 & 0.9192 & 0.9380 & 0.9312 & 0.8934 & 0.9537 \\
7 & 0.9149 & 0.9510 & 0.9379 & 0.9140 & 0.9516 \\
8 & 0.9310 & 0.9368 & 0.9347 & 0.8944 & 0.9597 \\
9 & 0.9346 & 0.9485 & 0.9434 & 0.9110 & 0.9628 \\
10 & 0.9576 & 0.9375 & 0.9448 & 0.8979 & 0.9751 \\
11 & 0.9169 & 0.9402 & 0.9317 & 0.8990 & 0.9515 \\
12 & 0.8811 & 0.9510 & 0.9257 & 0.9110 & 0.9339 \\
\hline
\end{tabular}

with morphological operations. A visual as well as parametric evaluation of enhanced regions shows encouraging results. The parameters that have been used for evaluation are PSNR, AMBE, EC and AMEE. In the next step, for these regions, features based on cluster shape and cluster texture have been extracted. A new set of features, based on recursive subsampling method, has been used in combination with the other shape-based features. These features have been reduced with the DEFS method and
SVM has been used for classification based on these reduced features. The performance of the shape-based and texture-based features has been analysed. It has been concluded that a combination of shape-based and texturebased features performs better than either shape-based or texture-based features alone. It has been observed that hierarchical centroid-based features improve the classification performance of the system (sensitivity from $90.99 \%$ to $96.57 \%$, specificity from $83.48 \%$ to $89.57 \%$ and accuracy from $88.51 \%$ to $94.25 \%$ ). The overall accuracy of classification system is $94.25 \%$ and AUC is 0.9307 . The unique combination of features extracted has a correlation with the factors describing a distinction between malignant and benign cluster regions. The performance of the system is validated by comparing the performance parameters of the proposed system with earlier work of the other researchers. A substantial fraction of malignant cases is diagnosed correctly and thereby proposed methodology increases the probability of a correct diagnosis and thus helps in reducing the number of unnecessary biopsies. In continuation of this work, in future, emphasis will also be given on individual microcalcification features along with the cluster shape features to improve the performance of the system.

Table 12. Performance of different features.

\begin{tabular}{|c|c|c|c|c|c|c|}
\hline Features & Sensitivity & Specificity & Accuracy & PPV & NPV & $\begin{array}{l}\text { Youden's } \\
\text { index }\end{array}$ \\
\hline Shape (without hierarchical centroid) & 0.5279 & 0.8696 & 0.6408 & 0.8913 & 0.4762 & 0.3975 \\
\hline Haralick & 0.7210 & 0.7478 & 0.7299 & 0.8528 & 0.5695 & 0.4689 \\
\hline Shape (without hierarchical centroid), Haralick & 0.6481 & 0.8870 & 0.7270 & 0.9207 & 0.5543 & 0.5350 \\
\hline Texture (without ALBP) & 0.7940 & 0.8174 & 0.8017 & 0.8981 & 0.6620 & 0.6114 \\
\hline Shape (without hierarchical centroid), texture (without ALBP) & 0.8970 & 0.8261 & 0.8736 & 0.9127 & 0.7983 & 0.7231 \\
\hline Shape(without hierarchical centroid), texture & 0.9099 & 0.8348 & 0.8851 & 0.9177 & 0.8205 & 0.7447 \\
\hline All shape and all texture & 0.9657 & 0.8957 & 0.9425 & 0.9494 & 0.9279 & 0.8613 \\
\hline
\end{tabular}

Table 13. The proposed system in comparison with other classification systems.

\begin{tabular}{|c|c|c|c|c|c|c|c|c|}
\hline $\begin{array}{l}\text { Paper and } \\
\text { author }\end{array}$ & Year & Database & Features used & Classifier & $\begin{array}{c}\text { Sensitivity } \\
(\%)\end{array}$ & $\begin{array}{l}\text { Specificity } \\
(\%)\end{array}$ & $\begin{array}{c}\text { Accuracy } \\
(\%)\end{array}$ & AUC \\
\hline $\begin{array}{l}\text { Karahaliou } \\
\quad \text { et al }\end{array}$ & 2007 & DDSM & Textural & $\mathrm{KNN}$ & 94.40 & 80.00 & & \\
\hline D'Aloia et al & 2013 & $\begin{array}{c}\text { MIAS and } \\
\text { DDSM }\end{array}$ & Cluster shape & SVM & 93.50 & & 94.20 & \\
\hline Strange et al & 2014 & DDSM & $\begin{array}{l}\text { Discrete } \\
\text { mereotopology }\end{array}$ & $\begin{array}{c}\text { Mereotopological } \\
\text { barcodes }\end{array}$ & & & 80.00 & 0.82 \\
\hline Chen et al & 2015 & DDSM & Graph theoretical & KNN & & & 86.00 & 0.91 \\
\hline $\begin{array}{l}\text { Proposed } \\
\text { method }\end{array}$ & 2016 & DDSM & Shape and texture & SVM & 96.57 & 89.57 & 94.25 & 0.93 \\
\hline
\end{tabular}




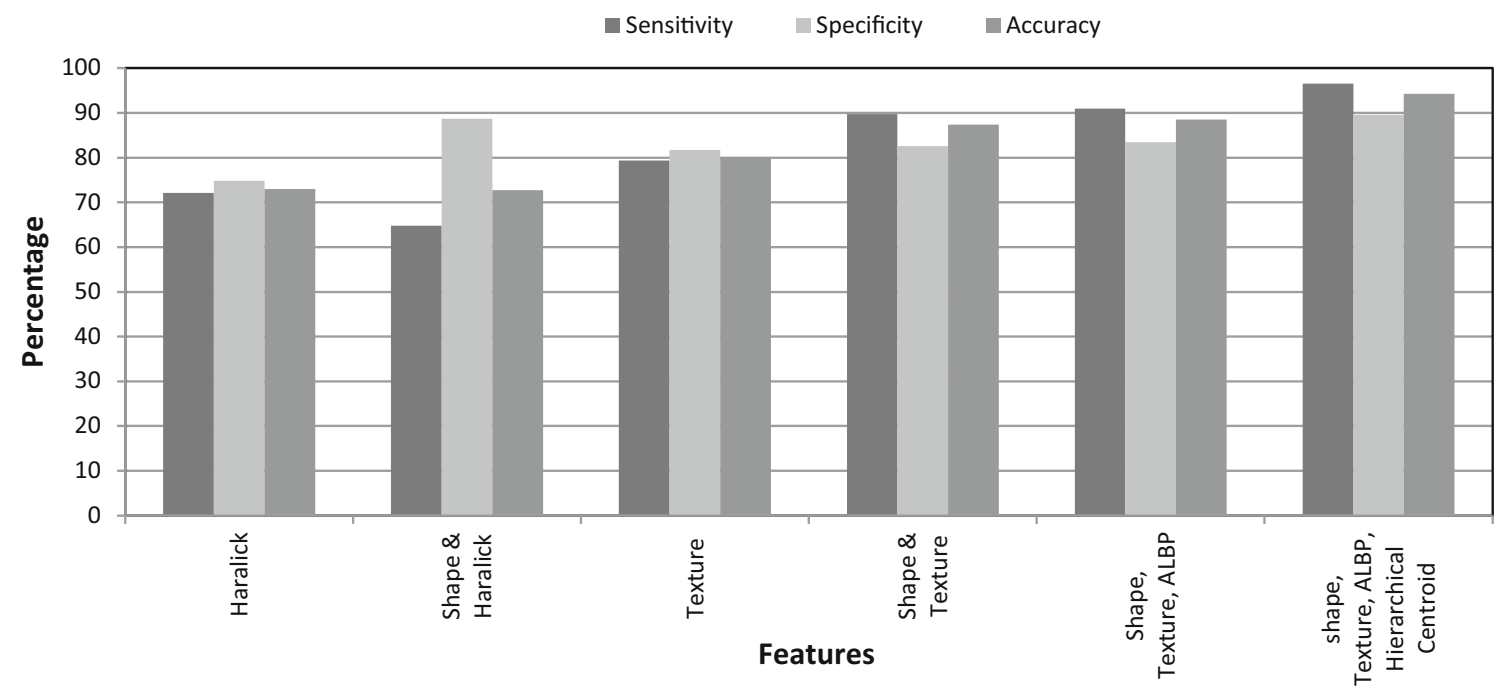

Figure 8. Contribution of the proposed features for the system.

\section{Acknowledgement}

Our thanks to Dr. Thomas Deserno, Department of Medical Informatics, Aachen University of Technology, Aachen, North Rhine-Westphalia, Germany, for providing the IRMA (Image Retrieval in Medical Applications) version of DDSM Database.

\section{References}

[1] Acharya U R, Ng E Y K, Tan J H and Sree S V 2012 Thermography based breast cancer detection using texture features and support vector machine. J. Med. Syst. 36: 1503-1510

[2] Cheng H D, Shi X J, Min R, Hu L M, Cai X P and Du H N 2006 Approaches for automated detection and classification of masses in mammograms. Pattern Recogn. 39(4): 646-668

[3] Sree S V, Ng E Y K, Acharya U R and Faust O 2011 Breast imaging: a survey. World J. Clin. Oncol. 2(4): 171-178

[4] Papadopoulos A, Fotiadis D I and Likas A 2005 Characterization of clustered microcalcifications in digitized mammograms using neural networks and support vector machines. Artif. Intell. Med. 34(2): 141-150

[5] Ganesan K, Acharya U R, Chua C K, Min L C, Mathew B and Thomas A K 2013 Decision support system for breast cancer detection using mammograms. Proc. Inst. Mech. Eng. Part H: J. Eng. Med. 227(7): 721-732

[6] Tiedeu A, Daul C, Kentsop A, Graebling P and Wolf D 2012 Texture-based analysis of clustered microcalcifications detected on mammograms. Digit. Signal Process. 22(1): 124-132

[7] Muttarak M, Kongmebhol P and Sukhamwang N 2009 Breast calcifications: which are malignant? Singapore Med. J. 50(9): 907-913

[8] Wei L, Yang Y, Nishikawa R M and Jiang Y 2005 A study on several machine learning methods for classification of malignant and benign clustered microcalcifications. IEEE Trans. Med. Imag. 24(3): 371-380
[9] Shanthi S and Bhaskaran V M 2014 A novel approach for classification of abnormalities in digitized mammograms. Sadhana 39(5): 1141-1150

[10] Karahaliou A, Skiadopoulos S, Boniatis I, Sakellaropoulos P, Likaki E, Panayiotakis G and Costaridou L 2007 Texture analysis of tissue surrounding microcalcifications on mammograms for breast cancer diagnosis. Br. J. Radiol. 80(956): 648-656

[11] Hamdi N, Auhmani K and Hassani M M R 2008 Design of a high-accuracy classifier based on Fisher discriminant analysis: application to computer-aided diagnosis of microcalcifications. In: Proceedings of the IEEE International Conference on Computational Sciences and its Applications (ICCSA'08), June 30-July 3, Perugia, pp. 267-273

[12] Tirtajaya A and Santika D D 2010 Classification of microcalcification using dual-tree complex wavelet transform and support vector machine. In: Proceedings of the IEEE $2^{\text {nd }}$ International Conference on Advances in Computing, Control and Telecommunication Technologies (ACT 2010), 2-3 December, Jakarta, pp. 164-166

[13] Zyout I and Abdel-Qader I 2011 Classification of microcalcification clusters via PSO-KNN Heuristic parameter selection and GLCM features. Int. J. Comput. Appl. 31(2): 34-39

[14] Farzana N and Rathnakara S 2011 Detection of micro-calcifications in mammogram images using probabilistic neural network. Elixir Comput. Sci. Eng. 38: 4404-4408

[15] Bhanumathi R and Suresh G R 2013 Detection of microcalcification in mammogram images using support vector machine based classifier. ITSI Trans. Electr. Electron. Eng. 1(2): 27-32

[16] Jenifer S, Parasuraman S and Kadirvel A 2014 An efficient biomedical imaging technique for automatic detection of abnormalities in digital mammograms. J. Med. Imag. Health Informat. 4(2): 291-296

[17] Strange H, Chen Z, Denton E R and Zwiggelaar R 2014 Modelling mammographic microcalcification clusters using persistent mereotopology. Pattern Recogn. Lett. 47: 157-163

[18] Lee S K, Chung P C, Chang C I, Lo C S, Lee T, Hsu G C and Yang C W 2003 Classification of clustered 
microcalcifications using a shape cognitron neural network. Neural Netw. 16(1): 121-132

[19] Soltanian-Zadeh H, Rafiee-Rad F and Pourabdollah-Nejad D S 2004 Comparison of multiwavelet, wavelet, Haralick, and shape features for microcalcification classification in mammograms. Pattern Recogn. 37(10): 1973-1986

[20] D'Aloia M, Rizzi M and Bari P D 2013 A second opinion system for microcalcification diagnosis. World Appl. Sci. J. 23(3): 289-295

[21] Karssemeijer N 1993 Recognition of microcalcification clusters in mammograms. Int. J. Pattern Recogn. Artif. Intell. 7(6): 1357-1376

[22] Zhang L, Qian W, Sankar R, Song D and Clark R 2001 A new false positive reduction method for MCCs detection in digital mammography. In: Proceedings of the IEEE International Conference on Acoustics, Speech and Signal Processing, 7-11 May, Salt Lake City, UT, pp. 1033-1036

[23] Cordella L P, Tortorella F and Vento M 2000 Combing experts with different features for classifying clustered microcalcifications in mammograms. In: Proceedings of the IEEE $15^{\text {th }}$ International Conference on Patten Recognition, 3-7 September, Barcelona, Spain, pp. 324-327

[24] De Oliveira J E, Deserno T M and Araújo A D A 2008 Breast lesions classification applied to a reference database. In: Proceedings of the $2^{\text {nd }}$ International Conference on E-Medical Systems, 29-31 October, Tunisia, pp. 1-7

[25] Kimori Y 2011 Mathematical morphology-based approach to the enhancement of morphological features in medical images. J. Clin. Bioinformat. 1(1): 1-10

[26] Stojić T and Reljin B 2010 Enhancement of microcalcifications in digitized mammograms: multifractal and mathematical morphology approach. FME Trans. 38(1): 1-9

[27] Vega-Corona A, Quintanilla-Domínguez J, Ojeda-Magaña B, Cortina-Januchs M G, Marcano-Cedeño A, Ruelas R and Andina D 2011 Microcalcifications detection using PFCM and ANN. In: Pattern Recognition. Berlin-Heidelberg: Springer, pp. 260-268

[28] Mustra M, Grgic M and Delac K 2012 Enhancement of microcalcifications in digital mammograms. In: Proceedings of the IEEE $19^{\text {th }}$ International Conference on Systems, Signals and Image Processing (IWSSIP), 11-13 April, Vienna, pp. 248-251

[29] Zhang X, Homma N, Goto S, Kawasumi Y, Ishibashi T, Abe M, Sugita N and Yoshizawa M 2013 A hybrid image filtering method for computer-aided detection of microcalcification clusters in mammograms. J. Med. Eng. Article ID 615254, pp. 1-8

[30] Quintanilla-Dominguez J, Ojeda-Magana B, Cortina-Januchs M C, Ruelas R, Vega-Corona A and Andina D 2011 Image segmentation by fuzzy and possibilistic clustering algorithms for identification of microcalcifications. Sci. Iran. 18(3): 580-589

[31] Nishikawa R M, Jiang Y, Giger M L, Doi K, Vyborny C J and Schmidt R A 1992 Computer-aided detection of clustered microcalcifications. In: Proceedings of the IEEE International Conference on Systems, Man and Cybernetics, 18-21 October, Chicago, pp. 1375-1378

[32] Mossi J M and Albiol A 1999 Improving detection of clustered microcalcifications using morphological connected operators. In: Proceedings of the IEEE $7^{\text {th }}$ International Conference on Image Processing and its Applications, 13-15 July, Manchester, pp. 498-501
[33] Pereira Jr R R, Marques P M A, Honda M O, Kinoshita S K, Engelmann R, Muramatsu C and Doi K 2007 Usefulness of texture analysis for computerized classification of breast lesions on mammograms. J. Digit. Imag. 20(3): 248-255

[34] Armon S 2011 Handwriting recognition and fast retrieval for Hebrew historical manuscripts. MTech Thesis, The Hebrew University of Jerusalem

[35] Vamvakas G, Gatos B and Perantonis S J 2010 Handwritten character recognition through two-stage foreground subsampling. Pattern Recogn. 43(8): 2807-2816

[36] Wu C M and Chen Y C 1992 Statistical feature matrix for texture analysis. CVGIP: Graph. Models Image Process. 54(5): 407-419

[37] Haralick R M, Shanmugam K and Dinstein I 1973 Textural features for image classification. IEEE Trans. Syst. Man Cybern. SMC-3: 610-621

[38] Laws K I 1980 Textured image segmentation. PhD Thesis, University of Southern California, Electrical Engineering

[39] Mavroforakis M E, Georgiou H V, Dimitropoulos N, Cavouras D and Theodoridis S 2006 Mammographic masses characterization based on localized texture and dataset fractal analysis using linear, neural and support vector machine classifiers. Artif. Intell. Med. 37(2): 145-162

[40] Wu C M, Chen Y C and Hsieh K S 1992 Texture features for classification of ultrasonic liver images. IEEE Trans. Med. Imag. 11(2): 141-152

[41] Guo Z, Zhang I, Zhang D and Zhang S 2010 Rotation invariant texture classification using adaptive LBP with directional statistical features. In: Proceedings of the IEEE $17^{\text {th }}$ International Conference on Image Processing, 26-29 September, Hong Kong, pp. 285-288

[42] Thangavel K and Velayutham C 2012 Rough set based unsupervised feature selection in mammogram image classification using entropy measure. J. Med. Imag. Health Informat. 2(3): 320-326

[43] Wang J and Zhao Y 2011 Differential evolution algorithm based one dimension real valued searching for feature selection. In: Future intelligent information systems. BerlinHeidelberg: Springer, 127-134

[44] Das S and Suganthan P N 2011 Differential evolution: a survey of the state-of-the-art. IEEE Trans. Evol. Comput. 15(1): 4-31

[45] Mala S and Latha K 2014 Feature selection in classification of eye movements using electrooculography for activity recognition. Comput. Math. Methods Med. Article ID 713818, pp. 1-9

[46] Bharathi P T and Subashini P 2014 Optimal feature subset selection using differential evolution and extreme learning machine. Int. J. Sci. Res. 3(7): 1898-1905

[47] Singh B, Jain V K and Singh S 2014 Mammogram mass classification using support vector machine with texture, shape features and hierarchical centroid method. J. Med. Imag. Health Inf. 4(5): 687-696

[48] Khushaba R N, Al-Ani A and Al-Jumaily A 2011 Feature subset selection using differential evolution and a statistical repair mechanism. Expert Syst. Appl. 38(9): 11515-11526

[49] Zyout I, Abdel-Qader I and Jacobs C 2009 Bayesian classifier with simplified learning phase for detecting microcalcifications in digital mammograms. Int. J. Biomed. Imag. Article ID 767805, pp. 1-13

[50] Bhateja V and Devi S 2010 Mammographic image enhancement using double sigmoid transformation function. 
In: Proceedings of the International Conference on Computer Applications (ICCA-2010), 24-27 December, Pondicherry, India, pp. 259-264

[51] Singh S, Yadav A and Singh B K 2011 Performance analysis of mammographic image enhancement techniques for early detection of breast cancer. In: Advances in parallel distributed computing. Berlin-Heidelberg: Springer, pp. 439-448

[52] Saleem A, Beghdadi A and Boashash B 2012 Image fusionbased contrast enhancement. EURASIP J. Image Video Process. 2012(1): 1-17

[53] Panetta K, Samani A and Agaian S 2014 Choosing the optimal spatial domain measure of enhancement for mammogram images. J. Biomed. Imag. Article ID 937849, pp. 1-8
[54] Gorgel P, Sertbas A, Kilic N and Ucan O N 2013 Mammographical mass detection and classification using local seed region growing-spherical wavelet transform hybrid scheme. Comput. Biol. Med. 43(6): 765-774

[55] Tang X, Ding H, Yuan Y E and Wang Q 2008 Morphological measurement of localized temperature increase amplitudes in breast infrared thermograms and its clinical application. Biomed. Signal Process. Control 3(4): 312-318

[56] Chen Z, Strange H, Oliver A, Denton E R, Boggis C and Zwiggelaar R 2015 Topological modeling and classification of mammographic microcalcification clusters. IEEE Trans. Biomed. Eng. 62(4): 1203-1214 